ARTICLE OPEN

\title{
Dissociable effects of acute SSRI (escitalopram) on executive, learning and emotional functions in healthy humans
}

\author{
Nikolina Skandali ${ }^{1,2,3}$, James B. Rowe ${ }^{2,4}$, Valerie Voon ${ }^{1,2,3}$, Julia B. Deakin ${ }^{1,3}$, Rudolf N. Cardinal (D) ${ }^{1,2,3}$, Francesca Cormack ${ }^{5}$, \\ Luca Passamonti ${ }^{4}$, William R. Bevan-Jones ${ }^{4}$, Ralf Regenthal $\mathbb{D}^{6}$, Samuel R. Chamberlain $\mathbb{D}^{1,3}$, Trevor W. Robbins ${ }^{2,7}$ and \\ Barbara J. Sahakian ${ }^{1,2}$
}

Serotonin is implicated in multiple executive functions including goal-directed learning, cognitive flexibility, response inhibition and emotional regulation. These functions are impaired in several psychiatric disorders, such as depression and obsessive-compulsive disorder. We tested the cognitive effects of the selective serotonin reuptake inhibitor escitalopram, using an acute and clinically relevant dose $(20 \mathrm{mg})$, in 66 healthy male and female volunteers in a double-blind, placebo-controlled study. Participants performed a cognitive test battery including a probabilistic and reversal learning task, the CANTAB intra-dimensional/extradimensional shift test of cognitive flexibility, a response inhibition task with interleaved stop-signal and No-Go trials and tasks measuring emotional processing. We showed that acute escitalopram administration impaired learning and cognitive flexibility, but improved the ability to inhibit responses in stop-signal trials while leaving unaffected acute emotional processing. Our findings suggest a dissociation of effects of acute escitalopram on cognitive functions, possibly mediated by differential modulation of brain serotonin levels in distinct functional neural circuits.

Neuropsychopharmacology (2018) 43:2645-2651; https://doi.org/10.1038/s41386-018-0229-z

\section{INTRODUCTION}

Serotonin (5-HT) is implicated in learning, executive and affective functions [1]. Much of the relevant evidence has depended on examining the effects of 5-HT loss in humans using acute dietary tryptophan depletion (ATD) [2], or in experimental animals, using 5-HT neurotoxins such as 5,7-dihydroxytryptamine (5,7-DHT) (e.g. [3]). ATD impairs visual discrimination learning and reversal [4], while selective $5-\mathrm{HT}$ depletion in the amygdala has been shown to increase sensitivity to aversive feedback in probabilistic learning and reversal tasks [5]. This is analogous to the effects of an acute low dose of citalopram in healthy volunteers [6] and rats [7] hypothesised to arise from inhibitory effects on 5-HT transmission [8]. However, higher doses or sub-chronic treatment with citalopram improve performance in probabilistic reversal learning tasks in rats [7]. These findings have clinical implications, as depressed humans show deficits in cognitive flexibility [9] and exaggerated reactions to negative feedback mediated by the prefrontal cortex (PFC) and the amygdala [10]. Moreover, selective serotonin reuptake inhibitors (SSRIs) constitute the firstline treatment for mood disorders [11].

This study examined the effects of acute escitalopram in healthy human volunteers on a test battery used extensively in both human and animal studies, assessing fronto-executive functions and previously shown to be sensitive to serotonergic manipulations $[6,12]$. We included (i) a probabilistic and reversal learning task; (ii) the CANTAB Intra-dimensional/Extra-dimensional set shift test, which includes reversal learning components; (iii) a combined
Stop-signal/Go/No-Go response inhibition task that measures both restraint and cancellation types of response inhibition in the same subjects [13] and (iv) emotional processing and social cognition tasks including the CANTAB affective Go/No-Go task, and the face affective Go/No-Go task and social information preference task, both from the newly developed EMOTICOM test battery [14]. Detailed hypotheses are described in the Methods section. An additional important feature of this study was its relatively novel examination of possible influences of gender, depressive symptoms and trait anxiety on the effects of escitalopram.

\section{MATERIALS AND METHODS}

Participants

The study was jointly sponsored by the University of Cambridge and Cambridgeshire and Peterborough NHS Foundation Trust and approved by the NHS East of England-Cambridge Central Research Ethics Committee (REC reference: 15/EE/0004). All participants were provided with verbal and written information on the study and gave written consent. Exclusion criteria included current or past psychiatric symptoms using a clinical structured interview (MINI International Neuropsychiatric Interview; [15]), personal or family history of psychiatric and neurological diseases, significant active or past medical problems, alcohol or drug abuse and excessive nicotine consumption (details in Supplementary Materials and Methods). Blood samples were collected at 2.5 and $5.5 \mathrm{~h}$ after drug administration.

\footnotetext{
${ }^{1}$ Department of Psychiatry, University of Cambridge, Cambridge, UK; ${ }^{2}$ Medical Research Council/Wellcome Trust Behavioural and Clinical Neuroscience Institute, University of

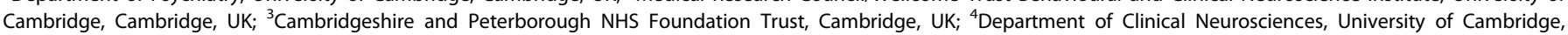

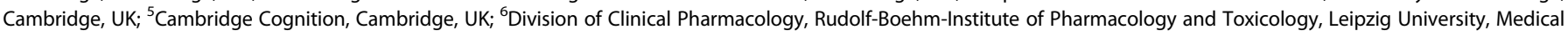
Faculty, Leipzig, Germany and ${ }^{7}$ Department of Psychology, University of Cambridge, Cambridge, UK Correspondence: Nikolina Skandali (ns594@cam.ac.uk)
}

Received: 7 June 2018 Revised: 24 August 2018 Accepted: 16 September 2018 Published online: 26 September 2018 


\section{Study design}

A double-blind, parallel-groups design was employed to eliminate effects of extensive training in learning tasks [16]. We administered escitalopram $20 \mathrm{mg}$, equivalent to $40 \mathrm{mg}$ of the racemic compound citalopram [17], as the activity of citalopram lies primarily on the S-enantiomer (escitalopram) and acute high dose of escitalopram was shown to alter electrophysiological measures of information processing [18].

Mood state and personality trait questionnaires

Participants were assessed for changes in mood state and drug side effects by completing computerised visual analogue scales at three time points. Participants also completed well-validated mood state and personality trait questionnaires, including the Beck Depression Inventory (BDI) [19] and State-Trait Anxiety Inventory (STAI) [20].

\section{Neuropsychological testing}

Neuropsychological testing started $3 \mathrm{~h}$ following pill administration to achieve peak plasma escitalopram concentrations [21]. Overall, 65 healthy volunteers completed the testing session (placebo; $N=33$, escitalopram; $N=32$ ) as one participant experienced side effects. Groups were matched demographically and in terms of baseline mood (Table 1).

\section{Statistical analysis}

Data were analysed with SPSS software, version 23.0. Appropriate statistical tests were applied, including chi-square test, two-tailed $t$ tests and ANOVAs, based on a priori hypotheses. Due to the number of tests administered, the Benjamini-Hochberg procedure was applied [22] for false discovery rate control (i.e. controlling the number of 'false positives' in our results) set a priori [23] at $q<0.15$ [24]. The false discovery rate assumes positive dependence or independence among variables, as was the case in our data, and it was performed across all hypotheses tested to provide a strict control of the false discovery rate. The Benjamini and Hochberg corrected significance level was 0.018 .

\section{Task description}

1. Probabilistic reversal learning task [6]: Participants made a twoalternative forced choice between two patterns (green and red) over a series of trials by touching the screen. Feedback was presented on the screen after each choice as 'CORRECT' and "INCORRECT". The pattern chosen in the first trial of the first stage was the correct one and participants received a ratio of 80:20 of accurate:misleading feedback for this in the first stage. Then the stimulus-outcome contingencies reversed (Figure S1 in Supplementary Materials and Methods).

\begin{tabular}{|llll|}
\hline Table 1. & Group demographics & \\
\hline Measures & $\begin{array}{l}\text { Placebo } \\
\text { group }\end{array}$ & Escitalopram group & $\begin{array}{l}\text { Group } \\
\text { difference }\end{array}$ \\
\hline Male:female & $17: 16$ & $16: 16$ & $p=0.903$ \\
Age & $25(5)$ & $27(7)$ & $p=0.061$ \\
NART & $42.45(4.93)$ & $42.9(5.27)$ & $p=0.723$ \\
Years of & $16.6(2.7)$ & $16.9(2.5)$ & $p=0.693$ \\
education & $35.3(8.6)$ & $34.6(9.5)$ & $p=0.77$ \\
$\begin{array}{l}\text { STAI trait anxiety } \\
\text { score }\end{array}$ & & & $p=0.32$ \\
BDI score & $5.3(5.1)$ & $4.1(4.4)$ & \\
\hline $\begin{array}{l}\text { Mean (SD) } \\
\text { aGroup difference: } p \text {-values of chi-square test for gender and two-tailed } t \\
\text { tests for the other measures }\end{array}$ & & \\
\hline
\end{tabular}

Primary outcome measures, defined as per Chamberlain et al. [6], were the number of errors until reaching learning criterion (eight correct consecutive responses to the most rewarding stimulus) in Stage 1 (indicating ability to encode the stimulus-outcome contingencies) and Stage 2 (indicating ability to learn the new stimulus-outcome contingencies). Secondary outcome measures included probabilities of win (reward)-stay/lose (no-reward)-shift strategy for each stage defined as per Rygula et al. [5]. Based on Chamberlain et al. [6], we hypothesised that the escitalopramtreated group would perform more errors during the probabilistic learning and after reversal and would show higher sensitivity to misleading feedback.

2. CANTAB Intra-Extra dimensional set shift task [25]: This is a nine-stage task consisting of visual discrimination, attention set formation and rule acquisition, maintenance of attention, setshifting and flexibility of attention and rule reversal. Primary measures [26] included errors in the critical stages of intradimensional set shift (IDS) (requiring the ability to apply a rule to new stimuli) and intra-dimensional shift reversal (IDR), extradimensional set shift (EDS) (requiring the ability to redirect attention towards a previously irrelevant dimension) and extradimensional shift reversal (EDR). Secondary measures included errors and response latencies in all stages.

3. Response inhibition task; the integrated Stop-signal and NoGo trials paradigm [27]: Participants were presented with three types of trials; 360 Go trials (75\%) requiring a right or left button press depending on the direction of a black arrow on the screen, 80 Stop-signal trials (17\%, with $\sim 50 \%$ successful) requiring cancellation of a cued button press when the black arrow turns red and 40 No-Go trials (8\%) requiring withholding themselves from pressing any button as the arrow appears red (Figure S2 in Supplementary Materials and Methods). Primary outcome measure was the estimated Stop-signal reaction time (SSRT) (i.e. the time required to abort an initiated action in the presence of a stop-signal) defined as per Ye et al. [27].

4. Emotional processing tasks: EMOTICOM face affective Go/NoGo task [14]: Participants are requested to press space bar in response to a specific target emotion when presented with blocks of facial stimuli (happy, sad and neutral facial expressions) as targets and distractors. Affective response bias was calculated as the difference in reaction time (RT) between the happy target/sad distractor condition and the sad target/happy distractor condition as per Bland et al. [14]. CANTAB affective Go/No-Go task [28]: Participants need to make a button press when they see a word of a specific valence (e.g. positive) in a series of blocks of two words with distinct emotional valence (positive, negative and neutral). Affective response bias was calculated as the difference in correct RTs between positive and negative blocks as per Murphy et al. [28]. EMOTICOM social information preference ('Theory of mind') task [14]: Participants are presented with socially ambiguous scenarios with pieces of information hidden from view, including three faces (revealing feelings), three thoughts and three facts. They select up to four pieces of information and then choose between three possible outcomes of neutral, positive or negative valence. Interpretational affective bias was calculated as the difference in the proportion of selected positive and negative scenario outcomes as per Bland et al. [14].

Study hypotheses

We hypothesised, based on prior literature on serotonergic interventions (Table S1 in Supplementary Materials and Methods), that participants receiving an acute escitalopram dosage of $20 \mathrm{mg}$ will make more errors during both probabilistic learning and after reversal of stimulus reward contingencies (perseverative errors) [6]. We predicted no effect or possible improvement [27] on tests of action restraint or cancellation, as previous studies applying Stop-signal paradigms produced mixed results and no behavioural effect on No-Go responding. Deficits were expected in tests of 


\begin{tabular}{|c|c|c|c|c|c|}
\hline & Measure & & Placebo & Escitalopram & Group difference $^{a}$ \\
\hline & RTs (ms) & & $2016.06(937.32)$ & 2165.79 (968.95) & $p=0.532$ \\
\hline & $\begin{array}{l}\text { Feedback type } \\
80 \% \text { accurate } \\
20 \% \text { misleading }\end{array}$ & & & & \\
\hline \multirow{5}{*}{ Probabilities } & & $20 \%$ & 1.14 & 4.03 & $p=0.104$ \\
\hline & Reward-shift & $80 \%$ & 5.97 & 9.48 & $p=0.125$ \\
\hline & & $20 \%$ & 4.92 & 8.47 & $p=0.279$ \\
\hline & Lose-stay & $80 \%$ & 1.42 & 4.13 & $p=0.125$ \\
\hline & & $20 \%$ & 88.64 & 72.18 & $\boldsymbol{p}=0.009$ \\
\hline \multirow{2}{*}{ Stage 2} & $\mathrm{RTs}(\mathrm{ms})$ & & $2251.86(6285.86)$ & $1280.32(865.23)$ & $p=0.237$ \\
\hline & $\begin{array}{l}\text { Feedback type } \\
80 \% \text { accurate } \\
20 \% \text { misleading }\end{array}$ & & & & \\
\hline \multirow[t]{7}{*}{ Probabilities } & Reward-stay & $80 \%$ & 68.75 & 63.71 & $p=0.376$ \\
\hline & & $20 \%$ & 11.74 & 15.32 & $p=0.359$ \\
\hline & Reward-shift & $80 \%$ & 6.06 & 8.57 & $p=0.238$ \\
\hline & & $20 \%$ & 4.92 & 6.58 & $p=0.442$ \\
\hline & Lose-stay & $80 \%$ & 16.1 & 16.32 & $p=0.237$ \\
\hline & & $20 \%$ & 70.08 & 62.9 & $p=0.971$ \\
\hline & Lose-shift & $80 \%$ & 10.07 & 12.6 & $p=0.684$ \\
\hline
\end{tabular}

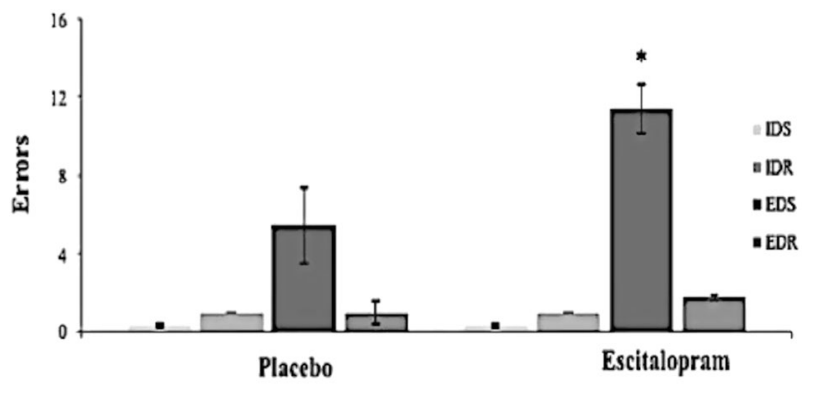

Treatment

Fig. 1 Escitalopram group made significantly more errors in the EDS, compared with the placebo group, following false discovery rate control of $q<0.15$ with the Benjamini-Hochberg procedure, as denoted with an asterisk

cognitive flexibility (although predominantly after reversal). We also hypothesised that acute escitalopram would influence affective bias in the three emotional processing tasks, as negative affective biases are well-documented in major depression [29] and following ATD in healthy volunteers [28], and acute administration of a clinically relevant dosage of citalopram in healthy volunteers was previously shown to increase recognition of happy faces and attention to socially relevant stimuli [30].

\section{RESULTS}

Biochemical analysis (placebo; $N=29$, escitalopram; $N=30$ )

Mean escitalopram plasma concentration at $2.5 \mathrm{~h}$ was $14 \mathrm{ng} / \mathrm{ml}$ (SD: $5.72, p<0.001, \mathrm{t}(54)=18.835)$, and at $5.5 \mathrm{~h}$ was $17.24 \mathrm{ng} / \mathrm{ml}$ (SD: $4.27, p<0.001, \mathrm{t}(54)=20.548$ ).

1. Probabilistic reversal learning task (placebo; $N=33$, escitalopram; $N=31$ ).

Stage 1 acquisition phase: The learning criterion was achieved by $26 / 31$ participants in the escitalopram group and 30/33 participants in the placebo group, $x^{2}=1.393, p=0.268$. However, the escitalopram group made significantly more errors, $U=314$, $z=-2.850, p=0.004$ (Table 2).

Stage 2 reversal phase: The learning criterion was achieved by $25 / 31$ participants in the escitalopram and 27/33 participants in the placebo groups, $X^{2}=0.014, p=0.904$. Errors and RTs were both similar between groups, $p>0.05$.

Win-stay/lose-shift: In Stage 1, a priori planned analyses showed that the escitalopram group had a significantly lower probability of win-stay, $p=0.043, \mathrm{~F}(1,62)=4.272$, partial $\eta^{2}=0.064$, and a significantly higher probability of lose-shift, $p=0.013, F(1,62)=$ 6.575 , partial $\eta^{2}=0.096$. We further assessed sensitivity to valid ( $80 \%$ of trials) or misleading ( $20 \%$ of trials) feedback by separating the above probabilities based on feedback type. Table 2 shows that the escitalopram group had a significantly higher probability of lose-shift after both misleading, $p=0.003, F(1,62)=9.724$, partial $\eta^{2}=0.136$, and accurate (valid) feedback, $p=0.032$, $\mathrm{F}(1,62)=4.834$, partial $\eta^{2}=0.72$. 
Table 3. Groups significantly differ in SSRT, but not in other measures in the response inhibition task

\begin{tabular}{llll}
\hline Measures (mean + SD) & Placebo & Escitalopram & Group difference ${ }^{\mathrm{a}}$ \\
\hline SSRT & $198.82(40.02)$ & $175.77(31.46)$ & $\boldsymbol{p}=0.013, \mathrm{t}(62)=-2.550$ \\
Go reaction time & $463.52(127.21)$ & $492.19(123.65)$ & $p=0.364, \mathrm{t}(62)=0.914$ \\
Go omission error rate & $0.005(0.01)$ & $0.01(0.04)$ & $p=0.179, \mathrm{t}(62)=1.361$ \\
Go commission error rate & $0.11(0.07)$ & $0.1(0.07)$ & $p=0.621, \mathrm{t}(62)=0.498$ \\
No-Go error rate & $0.11(0.14)$ & $0.08(0.14)$ & $p=0.459, \mathrm{t}(62)=0.745$ \\
\hline $\begin{array}{l}\text { Variables represent RTs in milliseconds } \\
\text { aGroup difference: } p \text {-value of independent samples } t \text { tests, significant results following false discovery rate control of } q<0.15 \text { with the Benjamini-Hochberg } \\
\text { procedure are denoted in bold }\end{array}$ \\
\hline
\end{tabular}

The two groups did not differ in win-shift behaviour after accurate (valid) feedback, $p>0.05$. There was no difference in either of these measures during Stage 2.

2. CANTAB Intra-Extra dimensional (ID/ED) set shift task (placebo; $N=31$, escitalopram; $N=30$ ): Repeated measures ANOVA for errors in the four critical stages of IDS, IDR, EDS and EDR showed a significant stage by treatment effect, $p=0.026, F(3,52)=3.339$, partial $\eta^{2}=0.162$. Both groups made fewer errors at the IDS compared with the EDS stage, $p=0.017, \mathrm{~F}(1,54)=6.091$, partial $\eta^{2}=$ 0.101 . Follow-up, one-way ANOVAs planned a priori for the IDS and EDS stages showed that the escitalopram group made significantly more errors at the EDS stage (mean: 11.4, SD: 10.49) than the placebo (mean: 5.43, SD: 6.93), $p=0.014, \mathrm{~F}(1,56)=6.449$, partial $\eta^{2}=0.103$ (Fig. 1). Escitalopram did not affect IDS errors, $p=0.561$. Follow-up, one-way ANOVAs for the reversal stages, IDR and EDR, also showed that escitalopram group made more errors at EDR, $p=0.082$, $F(1,59)=3.135$, partial $\eta^{2}=0.05$, as shown in Fig. 1 . The escitalopram group responded more slowly compared with the placebo in the EDS stage, $p=0.026, \mathrm{t}(59)=-2.307$, partial $\eta^{2}=0.102$. There was no effect on errors or RTs in the learning stages 1-5, $p>0.05$ (Table S2 in Supplementary Materials and Methods).

3. Response inhibition task (placebo; $N=33$, escitalopram; $N=32$ ): One participant outlier in the SSRT ( $>2$ SD from group mean) was excluded. Table 3 shows that escitalopram significantly reduced SSRT (speeded response inhibition), $p=0.013, \mathrm{t}(62)=-2.550$, without significant changes in other measures.

4. Effects of acute escitalopram on emotional processing: Table 4 shows that acute escitalopram administration did not alter affective bias in the three tasks, but did increase the proportion of facts chosen in the EMOTICOM social information preference task, $p=0.017, \mathrm{t}=2.451$, partial $\eta^{2}=0.095$, as shown with pre-planned independent samples $t$ tests.

Subjective measures and effects of trait anxiety, BDI and sex We found no interaction between treatment, and trait anxiety, BDI or sex in any task measure ( $p>0.05$ for all). Escitalopram had no significant effects on most subjective mood ratings $(p>0.05)$ (Table S3 in Supplementary Materials and Methods). The drug did enhance self-reported excitement $\left(p=0.028, \mathrm{~F}(1,29)=5.368\right.$, partial $\eta^{2}=0.156$, $p>0.05)$, but this was no longer present by the end of the testing session and did not contribute to any of the observed effects on the cognitive tests, following its inclusion as a covariate.

Variations in plasma escitalopram levels within the escitalopram group did not predict treatment effects on any of the aforementioned task measures ( $p>0.05$ for all).

\section{DISCUSSION}

This study shows reliable effects of an acute oral dose of the serotonin reuptake inhibitor escitalopram on cognitive as well as emotional functioning in healthy volunteers. Acute escitalopram impaired learning with uncertain reinforcement and enhanced responsivity to misleading negative feedback, analogous to that observed in depression. It produced dissociable effects on different aspects of executive function, specifically improving inhibitory response control (shorter SSRT), but impairing cognitive flexibility (impaired EDS performance). These differences reflect dissociable effects on functionally distinct aspects of executive function [31], leaving unaffected emotional processing at the group level, in healthy participants. These novel findings of acute effects of serotonin reuptake inhibition on cognitive performance have implications for understanding how central 5-HT pathways modulate cognition in health and psychiatric disorders.

Effects on probabilistic and deterministic learning

Healthy adults on escitalopram made more errors to criterion during Stage 1 of the probabilistic learning task, similar to effects of citalopram [6]. Further analysis revealed that escitalopram increased lose-shifting after misleading negative feedback, a significant finding after controlling for false discovery rate. In other words, there was a detrimental effect reminiscent of patients with depressive disorders [10] and effects in rats treated acutely with low doses of citalopram or forebrain 5-HT depletion [7]. Escitalopram increased lose-shifting after accurate feedback, but this finding did not survive significance when controlling for false discovery rate. There was no overall tendency to shift regardless of reinforcing feedback, as win-shift responding was not changed. These effects contrast with those reported for this task using ATD by Murphy et al. [28].

At the early stages of learning in the CANTAB ID/ED shifting paradigm, there were no effects of escitalopram, contrasting with effects of ATD (e.g. [12, 32]). The relative lack of sensitivity of the latter visual discrimination-learning task may relate to its deterministic nature, whereas the probabilistic learning task entails greater uncertainty.

Effects on inhibition and attention control

Acute escitalopram speeded SSRT. This cannot simply be attributed to strategic changes in responding as Go RT and other measures were unchanged. ATD has no significant effect on SSRT performance [33], although ATD can speed responding on punished Go trials of a Go/No-Go paradigm [34]. Whilst sharing many neurobehavioral processes of inhibitory response control, including the engagement of fronto-striatal 'loops' involving the right inferior frontal gyrus (BA 44 and 45$)[35,36]$, the Stop-signal and Go/No-Go paradigms appear to have some distinct underlying mechanisms [13]. Blockade of 5-HT reuptake with citalopram produced no effect on SSRT over a range of doses in rats $[13,37]$. In humans, citalopram $(30 \mathrm{mg})$ administration in healthy volunteers similarly produced no effect in a Stop-signal paradigm [6], although Ye et al. [27] showed that it improves SSRT and No-Go errors in Parkinson's disease patients with relatively severe symptoms.

The effects of serotonin on behavioural inhibition depend on the paradigm employed and action restraint differs from action cancellation [38, 39]. The SSRT might reflect a more sensitive (behavioural) measure than Go/No-Go performance-indeed the 
Table 4. Escitalopram effects on emotional processing tasks

\begin{tabular}{|c|c|c|c|c|}
\hline Task & & Escitalopram & Placebo & Group difference ${ }^{a}$ \\
\hline Stimulus type & Words & & & \\
\hline Sample size & $N=60$ (placebo; 31, escitalopram; 29) & & & \\
\hline \multirow[t]{2}{*}{ Measure } & Omission errors (shift blocks) & $2.9(3.89)$ & $2.03(2.4)$ & $p=0.843$ \\
\hline & Omission errors (non-shift blocks) & $2.62(3.8)$ & $1.87(2.29)$ & $p=0.712$ \\
\hline \multicolumn{5}{|c|}{ EMOTICOM affective Go/No-Go task } \\
\hline Stimulus type & Faces & & & \\
\hline Target emotions & Happy, sad, neutral & & & \\
\hline Sample size & $N=64$ (placebo; 32, escitalopram; 32) & & & \\
\hline Measure & Percentage of correct 'hit' responses for target emotion & & & \\
\hline Stimulus type & Outcomes of socially ambiguous situations & & & \\
\hline Target emotions & Positive, negative, neutral & & & \\
\hline Sample size & $N=58$ (placebo; 32, escitalopram; 27$)$ & & & \\
\hline Measure & Affective bias in scenario outcome choices (mean value) & 2.78 & 1.91 & $p=0.235$ \\
\hline \multirow[t]{4}{*}{ Stimulus type } & Information type (faces, thoughts, facts) & & & \\
\hline & Proportion of facts (mean \pm SD) & $0.15(0.1)$ & $0.1(0.07)$ & $\boldsymbol{p}=0.017$ \\
\hline & Proportion of thoughts (mean $\pm S D$ ) & $0.27(0.11)$ & $0.29(0.1)$ & $p=0.330$ \\
\hline & Proportion of faces (mean \pm SD) & $0.58(0.12)$ & $0.61(0.1)$ & $p=0.346$ \\
\hline
\end{tabular}

study by Macoveanu et al. [40] failed to show any significant effects on behavioural measures (errors and reaction times) of intravenous (i.v.) citalopram $(20 \mathrm{mg} / \mathrm{h})$ on $\mathrm{Go} / \mathrm{No}-\mathrm{Go}$ or on a variant where an alternative response had to be made. Additionally, although Del-Ben et al. [39] showed enhanced activation of the lateral orbito-frontal cortex following citalopram i.v. $7.5 \mathrm{mg}$ administration, no behavioural effect was observed. The present study is the first to show improvements in inhibitory response control performance by an acute high-dosage SSRI in healthy volunteers. The effects of serotonin manipulations may also vary according to baseline characteristics. For example, the change in activation of right inferior frontal gyrus during No-Go responding following ATD depends on neocortical $5-\mathrm{HT}_{2 \mathrm{~A}}$ receptor binding [40]. However, none of the effects of escitalopram on inhibition in the current study were affected by trait anxiety, depression, impulsiveness or gender.

Acute escitalopram treatment in healthy adults impaired extradimensional shift performance. Studies on rats (e.g. [41, 42]) and marmoset monkeys (e.g. [43]) implicate 5-HT in deterministic reversal learning deficits, rather than ED-shifting, with ATD impairing reversal learning (errors) and lengthening response latencies at certain stages of reversal and ID-shifting [32]. However, the common deficits produced on probabilistic learning and ED-shifting here suggest effects of the drug on neural circuits including the right ventro-lateral PFC (BA47), as both of these tasks are associated with activations of this region [44]. Moreover, the pharmacological fMRI study of Del-Ben et al. [39] emphasised strong interactions between i.v. administration of the SSRI citalopram and different task-related activations of this region.
Effect on emotional processing

In contrast to its effects on learning and 'cold' executive performance, acute escitalopram had relatively little effect on emotional processing or so-called 'hot' cognition [45] (see Table 4), even taking into account trait anxiety, BDI and gender influences. Face recognition was measured in the EMOTICOM faces affective Go/No-Go task where participants had to respond to a target face ignoring the distractor, but Table 4 shows that escitalopram produced no effects on accurate 'hit' responses. The groups also did not differ in omission errors for distinct target valence words in the CANTAB affective Go/No-Go test-a task previously shown to be sensitive to ATD (enhancing negative affective bias) [29] and depression [28]. We did find a significant preference for subjects treated with escitalopram to use facts in preference to faces or thoughts to interpret ambiguous scenarios in the novel "Social Information Processing" task. This result suggests that informational aspects of socio-emotional processing used for decisionmaking may be diminished.

In relation to the published literature, Del-Ben et al. [39] found no behavioural effects on face recognition of i.v. citalopram (7.5 mg) treatment. Similarly, Murphy et al. [46] also found no behavioural effect on fear recognition of oral citalopram, although the BOLD response to fearful faces in the amygdala was reduced. Harmer et al. [30] and Browning et al. [47] did report enhanced recognition of both fear and happiness in faces by predominantly female healthy volunteers following acute citalopram (oral $20 \mathrm{mg}$ or iv. $10 \mathrm{mg}$ )-though decreased recognition of fear in faces following ATD in female volunteers [48]. It is possible that the effects of SSRIs on emotion are critically dependent on dose and 
plasma levels; our dose of escitalopram led to plasma levels comparable with the lower end of the clinical range. However, direct comparison with the studies above was not possible as plasma levels were not routinely reported. Of course, it is possible in theory that higher doses of acute escitalopram would have produced more robust effects on emotional processing. Overall however this study shows that behavioural measures of emotional processing are somewhat insensitive to acute SSRI treatment in comparison to more cognitive indices. Indeed, cognitive changes may contribute to emotional sequelae. For example, impaired cognitive flexibility, as shown here, may promote ruminative thinking that leads to anxiety. Such cognitive effects may even contribute to the initial increased anxiety that patients experience when initiating treatment [11]

\section{Implications for understanding 5-HT function: comparisons with} ATD

The inhibitory actions of acute escitalopram administration on terminal 5-HT release, through actions at auto-receptors on raphé 5-HT neurons [8], may be analogous to the presumed transient reduction in 5-HT activity caused by ATD [49]. Nord et al. [50] found that the same dose of escitalopram, as used here $(20 \mathrm{mg}$ oral), produced changes in PET ligand binding, suggestive of reduced 5 -HT in cortical (rather than subcortical) regions in male humans. Higher acute i.v. doses in female rhesus monkeys produced apparently increased 5-HT levels, particularly in cortical and thalamic regions presumably because auto-receptor effects are outweighed by greater effects at the synaptic terminals.

Acute versus chronic serotonin reuptake inhibition can have opposite functional effects on emotional processing and facets of information processing $[6,51]$. Whether these opposite actions are respectively due to globally diminished or enhanced 5-HT activity is still unclear. However, the same acute dose can also produce a mixture of apparently opposite functional effects in different domains, e.g. improved SSRT and worsened ED-shifting, and enhanced processing of both 'fearful' and 'happy' emotions [47]. This might arise because of differential effects of the SSRI in different brain regions sub-serving these functions, perhaps because of regional marked variations in SERT density [52] or in differences in inhibitory auto-receptors in the dorsal and median raphé nuclei [8], as well as their projections to the forebrain regions (to striatum, amygdala and neocortex and to hippocampus and limbic regions, respectively; [53]). The PET studies by Nord et al. [50] also suggest that they result from diminished 5-HT post-synaptic actions in certain regions, mainly in the neocortex. Alternatively, Del-Ben et al. [39] showed that acute oral citalopram could affect the same orbito-frontal/ventro-lateral PFC region differentially according to the task, as well as producing other region-specific changes in the functional BOLD response.

These observations make it plausible that global changes in 5HT function produced by escitalopram may have differential effects on cognitive and emotional processes mediated by different brain regions or networks. Another way of formulating this point is that comparable changes in 'arousal' produced by alterations in 5-HT function may have differential effects requiring different levels of 'arousal' for optimal performance.

Contrasting escitalopram with ATD, the latter may have very different effects on regional $5-\mathrm{HT}$ function, distinct from those produced by acute escitalopram [2]. Although there have been relatively few direct comparisons of these treatments using brain imaging methodology (e.g. [40]), evidently, acute escitalopram and ATD do not necessarily reflect opposite effects on 5-HT functioning.

Primary study limitations were the use of only one dose of escitalopram, although it was the clinically highest allowed one and higher acute doses being precluded by ethical considerations. A sub-chronic study of the effects of this drug is also now urgently indicated to determine to what extent these acute effects persist or even reverse. We employed a between-group design because of well-known practice effects confounding crossover designs on cognitive functions [16]; however, the groups were well matched. We employed a large number of tests of cognition and emotional function, but controlled for false discovery rate across the entire test battery.

\section{Summary}

We showed contrasting detrimental and enhancing effects of acute high-dose escitalopram on cognitive functioning in human volunteers, while probabilistic learning was impaired in a manner consistent with the effects of reduced 5-HT transmission and depression. The results are relevant to understanding the clinical effects of SSRIs at early stages of treatment, as well as the role of $5-\mathrm{HT}$ in emotional and cognitive processing.

\section{FUNDING AND DISCLOSURE}

This work was supported by a Wellcome Trust Senior Investigator Award to TW Robbins (104631/Z/14/Z) and the NIHR Cambridge Biomedical Research Centre (Mental Health theme). TWR consults for Cambridge Cognition, Mundipharma and Unilever have held research grants from Shionogi and Lundbeck and receives Royalties for CANTAB. NS was supported by a Medical Research Council Doctoral Grant Studentship (1432057), VV by a Medical Research Council Senior Fellowship (MR/P008747/1), SCR by a Wellcome Trust Clinical Fellowship (110049/Z/15/Z), LP by the Medical Research Council (MR/P01271X/1) and FC is employed by Cambridge Cognition. JBR is supported by the Wellcome Trust (103838) and received research grants unrelated to this work from Medical Research Council, James S McDonnell Foundation, PSPAssociation, AZ-Medimmune, Janssen and Lilly, and served as editor for Brain. RR received a grant (16KNO16238) from the Federal Ministry for Economic Affairs and Energy. BJS consults for Cambridge Cognition, PEAK and Mundipharma and SRC consults for Cambridge Cognition and Shire. JBD, RC and WR Bevan-Jones have nothing for disclosure.

\section{ACKNOWLEDGEMENTS}

We thank Dr. David Nesbitt for help with medical cover and Drs. Annemieke ApergisSchoute and Annette Bruhl for discussions.

\section{ADDITIONAL INFORMATION}

Supplementary Information accompanies this paper at (https://doi.org/10.1038/ s41386-018-0229-z).

\section{REFERENCES}

1. Cools R, Roberts AC, Robbins TW. Serotoninergic regulation of emotional and behavioural control processes. Trends Cogn Sci. 2008;12:31-40.

2. Faulkner $P$, Deakin JW. The role of serotonin in reward, punishment and behavioural inhibition in humans: insights from studies with acute tryptophan depletion. Neurosci \& Biobehav Rev. 2014;46:365-78.

3. Clarke HF, Dalley JW, Crofts HS, Robbins TW, Roberts AC. Cognitive inflexibility after prefrontal serotonin depletion. Science. 2004;304:878-80.

4. Rogers RD, Andrews TC, Grasby PM, Brooks DJ, Robbins TW. Contrasting cortical and subcortical activations produced by attentional-set shifting and reversal learning in humans. J Cogn Neurosci. 2000;12:142-62.

5. Rygula R, Clarke HF, Cardinal RN, Cockcroft GJ, Xia J, Dalley JW, et al. Role of central serotonin in anticipation of rewarding and punishing outcomes: effects of selective amygdala or orbitofrontal 5-HT depletion. Cereb Cortex. 2014;25: 3064-76.

6. Chamberlain SR, Müller U, Blackwell AD, Clark L, Robbins TW, Sahakian BJ. Neurochemical modulation of response inhibition and probabilistic learning in humans. Science. 2006;311:861-3.

7. Bari A, Theobald DE, Caprioli D, Mar AC, Aidoo-Micah A, Dalley JW, et al. Serotonin modulates sensitivity to reward and negative feedback in a probabilistic reversal learning task in rats. Neuropsychopharmacology. 2010;35:1290. 
8. Blier P, Serrano A, Scatton B. Differential responsiveness of the rat dorsal and median raphe $5-\mathrm{HT}$ systems to $5-\mathrm{HT} 1$ receptor agonists and p-chloroamphetamine. Synapse. 1990;5:120-33.

9. Dickstein DP, Finger EC, Brotman MA, Rich BA, Pine DS, Blair JR, et al. Impaired probabilistic reversal learning in youths with mood and anxiety disorders. Psychol Med. 2010:40:1089-1100.

10. Tavares JVT, Clark L, Furey ML, Williams GB, Sahakian BJ, Drevets WC. Neural basis of abnormal response to negative feedback in unmedicated mood disorders. Neuroimage. 2008;42:1118-26.

11. Taylor D, Paton C, Kapur S. The Maudsley prescribing guidelines in psychiatry, 12th edition, 2015.

12. Park SB, Coull JT, McShane RH, Young AH, Sahakian BJ, Robbins TW, et al. Tryptophan depletion in normal volunteers produces selective impairments in learning and memory. Neuropharmacology. 1994;33:575-88.

13. Eagle DM, Bari A, Robbins TW. The neuropsychopharmacology of action inhibition: cross-species translation of the stop-signal and go/no-go tasks. Psychopharmacol (Berl). 2008;199:439-56.

14. Bland AR, Roiser JP, Mehta MA, Schei T, Boland H, Campbell-Meiklejohn DK, et al. EMOTICOM: a neuropsychological test battery to evaluate emotion, motivation, impulsivity, and social cognition. Front Behav Neurosci. 2016;10:25.

15. Sheehan DV, Janavs J, Baker R, Harnett-Sheehan K, Knapp E, Sheehan M, et al. MINI-mini international neuropsychiatric interview-english version 5.0. 0-DSM-IV. J Clin Psychiatry. 1998;59:34-57.

16. Shaughnessy JJ, Zechmeister EB, Zechmeister JS. Research methods in psychology. 9th ed. New York, NY: McGraw-Hill; 2012.

17. Hayasaka $Y$, Purgato $M$, Magni LR, Ogawa $Y$, Takeshima N, Cipriani A, et al. Dose equivalents of antidepressants: Evidence-based recommendations from randomized controlled trials. J Affect Disord. 2015;180:179-84.

18. Wienberg M, Glenthoj BY, Jensen KS, Oranje B. A single high dose of escitalopram increases mismatch negativity without affecting processing negativity or P300 amplitude in healthy volunteers. J Psychopharmacol. 2010;24:1183-92.

19. Beck AT. Depression: causes and treatment. Philadelphia: University of Pennsylvania Press; 1972.

20. Spielberger CD, Gorsuch RL, Lushene RD, Vagg PR, Jacobs GA. Manual for the statetrait anxiety inventory (STAI). Palo Alto, CA: Consulting Psychologists Press; 1983.

21. Rao N. The clinical pharmacokinetics of escitalopram. Clin Pharmacokinet. 2007;46:281-90.

22. Benjamini $Y$, Hochberg $Y$. Controlling the false discovery rate: a practical and powerful approach to multiple testing. J R Stat Soc B. 1995;57:289-300.

23. McDonald JH. Handbook of biological statistics. 3rd ed. Baltimore, MD: Sparky House Publishing; 2014. p. 254-260. http://www.biostathandbook.com/.

24. Genovese CR, Lazar NA, Nichols T. Thresholding of statistical maps in functional neuroimaging using the false discovery rate. Neuroimage. 2002;15:870-8.

25. Sahakian BJ, Owen AM. Computerized assessment in neuropsychiatry using CANTAB: discussion paper. J R Soc Med. 1992;85:399.

26. Potter GG, McQuoid DR, Payne ME, Taylor WD, Steffens DC. Association of attentional shift and reversal learning to functional deficits in geriatric depression. Int J Geriatr Psychiatry. 2012;27:1172-9.

27. Ye Z, Altena E, Nombela C, Housden CR, Maxwell H, Rittman T, et al. Selective serotonin reuptake inhibition modulates response inhibition in Parkinson's disease. Brain. 2014;137:1145-55.

28. Murphy F, Smith K, Cowen P, Robbins T, Sahakian B. The effects of tryptophan depletion on cognitive and affective processing in healthy volunteers. Psychopharmacol (Berl). 2002;163:42-53.

29. Murphy FC, Sahakian BJ, Rubinsztein JS, Michael A, Rogers RD, Robbins TW, et al Emotional bias and inhibitory control processes in mania and depression. Psychol Med. 1999;29:1307-21.

30. Harmer CJ, Bhagwagar Z, Perrett DI, Vollm BA, Cowen PJ, Goodwin GM. Acute SSRI administration affects the processing of social cues in healthy volunteers. Neuropsychopharmacology. 2003a;28:148-52.

31. Friedman NP, Miyake A, Corley RP, Young SE, DeFries JC, Hewitt JK. Not all executive functions are related to intelligence. Psychol Sci. 2006;17:172-9.

32. Rogers RD, Blackshaw AJ, Middleton HC, Matthews K, Hawtin K, Crowley C, et al. Tryptophan depletion impairs stimulus-reward learning while methylphenidate disrupts attentional control in healthy young adults: implications for the monoaminergic basis of impulsive behaviour. Psychopharmacol (Berl). 1999;146:482-4

33. Clark L, Roiser JP, Cools R, Rubinsztein DC, Sahakian BJ, Robbins TW. Stop signal response inhibition is not modulated by tryptophan depletion or the serotonin transporter polymorphism in healthy volunteers: implications for the 5 - $\mathrm{HT}$ theory of impulsivity. Psychopharmacol (Berl). 2005;182:570-8.

34. Crockett MJ, Clark L, Robbins TW. Reconciling the role of serotonin in behavioral inhibition and aversion: acute tryptophan depletion abolishes punishmentinduced inhibition in humans. J Neurosci. 2009:29:11993-9.
35. Rae CL, Hughes LE, Weaver C, Anderson MC, Rowe JB. Selection and stopping in voluntary action: a meta-analysis and combined fMRI study. Neuroimage. 2014;86:381-91.

36. Rubia K, Lee F, Cleare AJ, Tunstall N, Fu CH, Brammer M, et al. Tryptophan depletion reduces right inferior prefrontal activation during response inhibition in fast, event-related fMRI. Psychopharmacol (Berl). 2005;179:791-803.

37. Bari A, Eagle DM, Mar AC, Robinson ES, Robbins TW. Dissociable effects of noradrenaline, dopamine, and serotonin uptake blockade on stop task performance in rats. Psychopharmacol (Berl). 2009;205:273-83.

38. Dalley JW, Robbins TW. Fractionating impulsivity: neuropsychiatric implications. Nat Rev Neurosci. 2017;18:158.

39. Del-Ben CM, Deakin JW, Mckie S, Delvai NA, Williams SR, Elliott R, et al. The effect of citalopram pretreatment on neuronal responses to neuropsychological tasks in normal volunteers: an FMRI study. Neuropsychopharmacology. 2005 30:1724.

40. Macoveanu J, Hornboll B, Elliott R, Erritzoe D, Paulson OB, Siebner H, et al. Serotonin $2 \mathrm{~A}$ receptors, citalopram and tryptophan-depletion: a multimodal imaging study of their interactions during response inhibition. Neuropsychopharmacology. 2013;38:996.

41. Barlow RL, Alsiö J, Jupp B, Rabinovich R, Shrestha S, Roberts AC, et al. Markers of serotonergic function in the orbitofrontal cortex and dorsal raphé nucleus predict individual variation in spatial-discrimination serial reversal learning. Neuropsychopharmacology. 2015;40:1619.

42. Danet M, Lapiz-Bluhm S, Morilak DA. A cognitive deficit induced in rats by chronic intermittent cold stress is reversed by chronic antidepressant treatment. Int $J$ Neuropsychopharmacol. 2010;13:997-1009.

43. Clarke HF, Walker SC, Crofts HS, Dalley JW, Robbins TW, Roberts AC. Prefrontal serotonin depletion affects reversal learning but not attentional set shifting. J Neurosci. 2005;25:532-8.

44. Cools R, Clark L, Owen AM, Robbins TW. Defining the neural mechanisms of probabilistic reversal learning using event-related functional magnetic resonance imaging. J Neurosci. 2002;22:4563-7.

45. Roiser JP, Sahakian BJ. Hot and cold cognition in depression. CNS Spectr. 2013;18:139-49.

46. Murphy SE, Norbury R, O'Sullivan U, Cowen PJ, Harmer CJ. Effect of a single dose of citalopram on amygdala response to emotional faces. $\mathrm{Br} J$ Psychiatry. 2009;194:535-40.

47. Browning M, Reid C, Cowen PJ, Goodwin GM, Harmer CJ. A single dose of citalopram increases fear recognition in healthy subjects. J Psychopharmacol. 2007;21:684-90.

48. Harmer CJ, Rogers RD, Tunbridge E, Cowen PJ, Goodwin GM. Tryptophan depletion decreases the recognition of fear in female volunteers. Psychopharmacol (Berl). 2003b;167:411-7.

49. Young SN. Acute tryptophan depletion in humans: a review of theoretical practical and ethical aspects. J Psychiatry \& Neurosci: JPN. 2013;38:294.

50. Nord M, Finnema SJ, Halldin C, Farde L. Effect of a single dose of escitalopram on serotonin concentration in the non-human and human primate brain. Int J Neuropsychopharmacol. 2013;16:1577-86.

51. Harmer CJ, Mackay CE, Reid CB, Cowen PJ, Goodwin GM. Antidepressant drug treatment modifies the neural processing of nonconscious threat cues. Biol Psychiatry. 2006;59:816-20.

52. Majuri J, Joutsa J, Johansson J, Voon V, Parkkola R, Alho H, et al. Serotonin transporter density in binge eating disorder and pathological gambling: a PET study with [11C] MADAM. Eur Neuropsychopharmacol. 2017;27:1281-8.

53. Hornung J-P. The Neuroanatomy of the Serotonergic System. In Handbook of the Behavioral Neurobiology of Serotonin. Handbook of Behavioral Neuroscience, Vol. 21. In: Muller CP, Jacobs BL, editors. Amsterdam: Elsevier; 2010. p. 51-64.

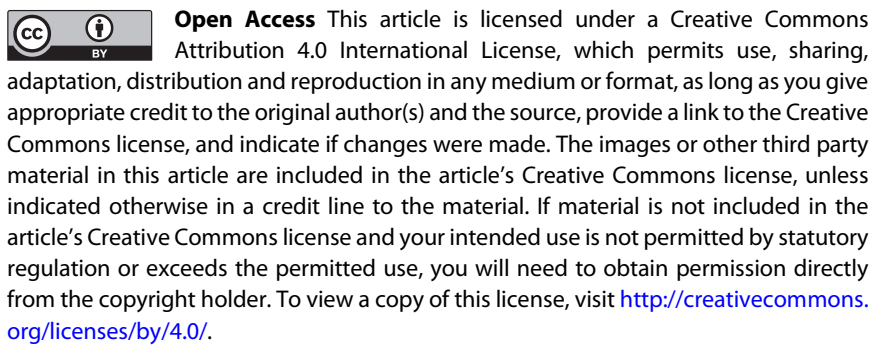

C) The Author(s) 2018 